\title{
SNARE and regulatory proteins induce local membrane protrusions to prime docked vesicles for fast calcium-triggered fusion
}

\author{
Tanmay A M Bharat ${ }^{1,3, \dagger}$, Jörg Malsam²,† ${ }^{2, W i m ~ J ~ H ~ H a g e n ~}{ }^{1}$, Andrea Scheutzow ${ }^{2}$, Thomas H Söllner ${ }^{2,{ }^{* *}}$ \& \\ John A G Briggs ${ }^{1, *}$
}

\begin{abstract}
Synaptic vesicles fuse with the plasma membrane in response to $\mathrm{Ca}^{2+}$ influx, thereby releasing neurotransmitters into the synaptic cleft. The protein machinery that mediates this process, consisting of soluble $\mathrm{N}$-ethylmaleimide-sensitive factor attachment protein receptors (SNAREs) and regulatory proteins, is well known, but the mechanisms by which these proteins prime synaptic membranes for fusion are debated. In this study, we applied large-scale, automated cryo-electron tomography to image an in vitro system that reconstitutes synaptic fusion. Our findings suggest that upon docking and priming of vesicles for fast $\mathrm{Ca}^{2+}$-triggered fusion, SNARE proteins act in concert with regulatory proteins to induce a local protrusion in the plasma membrane, directed towards the primed vesicle. The SNAREs and regulatory proteins thereby stabilize the membrane in a high-energy state from which the activation energy for fusion is profoundly reduced, allowing synchronous and instantaneous fusion upon release of the complexin clamp.
\end{abstract}

Keywords cryo-electron microscopy; cryo-electron tomography; in vitro reconstitution; membrane curvature; SNARE-mediated membrane fusion Subject Categories Membrane \& Intracellular Transport; Neuroscience DOI 10.1002/embr.201337807 | Received 24 July 2013 | Revised 18 December 2013 | Accepted 18 December 2013 | Published online 3 February 2014 EMBO Reports (2014) 15, 308-314

\section{Introduction}

In neurons, synaptic vesicles fuse with the plasma membrane within 100 microseconds of $\mathrm{Ca}^{2+}$ influx, causing near-instantaneous release of neurotransmitters into the synaptic cleft $[1,2]$. This requires that a readily releasable pool of synaptic vesicles is docked at the plasma membrane, in a state primed for fusion $[3,4]$. The core protein machinery for membrane fusion consists of the soluble $\mathrm{N}$-ethylmaleimide-sensitive factor attachment protein receptors (SNAREs), which are characterized by SNARE motifs: extended alphahelices that have the propensity to assemble together with cognate SNARE proteins into four-helix bundles [5,6]. The v-SNARE VAMP2 on synaptic vesicles interacts with its cognate t-SNARE syntaxin1/ SNAP-25 on the plasma membrane [7] to form a trans-SNARE complex. Trans-SNARE complex assembly occurs in a zipper-like manner beginning with the N-terminal, membrane-distal halves of the SNARE motifs, proceeding through the membrane proximal halves of the SNARE motifs, thereby bringing the membrane in close contact, and continuing through the linker into the transmembrane regions to form the fully-assembled post-fusion state $[8,9]$. This process suggests a model where SNARE complex assembly generates a mechanical force to induce membrane fusion. Dependent on the SNARE complex zippering state, increasing forces might be transmitted to the lipid bilayers, resulting in local changes to membrane shape.

Intermediate stages in assembly provide natural targets for regulatory proteins and the current model predicts [10-13] the following protein roles. Synaptotagmin 1 , the $\mathrm{Ca}^{2+}$ sensor, is an integral membrane protein in synaptic vesicles and binds the t-SNARE syntaxin1/ SNAP-25 on the plasma membrane, thereby docking vesicles [14-19]. Complexin stabilizes the N-terminal half of the partially assembled four-helix bundle, but blocks further zippering of the C-terminal half by occupying the membrane proximal region of syntaxin1/SNAP-25 [14,20,21]. The cytosolic SM (Sec1/Munc18-like) protein Munc18-1 binds the preassembled t-SNARE complex and the membrane proximal section of VAMP2 and thereby promotes further SNARE complex assembly [22,23], but does not overcome the complexin-induced arrest [14]. Upon $\mathrm{Ca}^{2+}$ influx via a voltage-gated $\mathrm{Ca}^{2+}$ channel, the $\mathrm{C} 2$ domains of synaptotagmin interact with anionic phospholipids and the t-SNARE, causing local phospholipid bilayer perturbation and the release of the complexin inhibition, triggering fusion $[13,24]$.

\footnotetext{
Structural and Computational Biology Unit, European Molecular Biology Laboratory, Heidelberg, Germany

Heidelberg University Biochemistry Center, Heidelberg, Germany

3 Structural Studies Division, MRC Laboratory of Molecular Biology, Cambridge, UK

*Corresponding author. Tel: +49 6221387 8482; Fax: +49 6221387 8519; E-mail: john.briggs@embl.de

${ }^{* *}$ Corresponding author. Tel: +49 622154 5342; Fax: +49 622154 5341; E-mail: thomas.soellner@bzh.uni-heidelberg.de

$\dagger$ These authors contributed equally to this work.
} 
Although the molecular machinery controlling membrane fusion has been identified, it is unclear how the network of SNAREs and regulatory components manipulates and constrains the lipid membranes to prime and mediate fusion. Two recent studies have addressed this question using cryo-electron microscopy (cryoEM) to image mixtures of reconstituted liposomes containing $\mathrm{V}$ - and t-SNAREs and a subset of regulatory proteins. The first study [25] was primarily limited to SNAREs and used a fragment of VAMP2 (syntaptobrevin) to slow down SNARE complex assembly and allow intermediates to be visualized. In this system, extended tight membrane contacts between liposomes were observed, some of which had undergone hemifusion, and it was proposed that fusion is induced at the edges of extended membrane interfaces. In the second study [26], small unilamellar vesicles (SUVs) contained reconstituted SNAREs and reconstituted full-length synaptotagmin 1. Hemifusion diaphragms were observed, as well as points where liposomes contacted one another but had undergone no change in membrane shape. No extended tight membrane contacts were observed in the absence of $\mathrm{Ca}^{2+}$. Upon $\mathrm{Ca}^{2+}$ addition, only the point-contacts underwent fast fusion.

Here, we have re-addressed how membranes are primed for fusion by imaging an in vitro reconstituted system mimicking synaptic vesicle fusion [14] in three dimensions by cryo-electron tomography (cryoET).

\section{Results and Discussion}

We mixed SUVs, into which we had inserted VAMP2 and synaptotagmin 1, with giant unilamellar vesicles (GUVs) containing syntaxin1 and SNAP-25. SNARE complex formation was promoted by Munc18-1, but stalled by the presence of complexin (Fig 1A, Supplementary Methods). Using a fluorescence dequenching assay we verified that $\mathrm{Ca}^{2+}$ triggers fast, synchronous lipid mixing in this system, in a manner dependent on the presence of the reconstituted SNAREs [14] (Fig 1B).

Reactions were incubated for $1 \mathrm{~min}$ at $37^{\circ} \mathrm{C}$ in the absence of $\mathrm{Ca}^{2+}$, vitrified by plunge-freezing, and transferred to the electron microscope for imaging (Supplementary Methods). We collected low-magnification overview images (Supplementary Fig S1) of large areas of the samples to assess the degree to which the SUVs were docked to the GUVs, and collected high-magnification cryo-electron tomograms of docked SUVs to obtain 3D views of the membrane morphology at the docking site. Reconstructed cryo-electron

A<smiles>O=C[14CH]=C[14CH]=O</smiles>

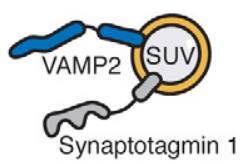
Membrane fusion arrested by complexin
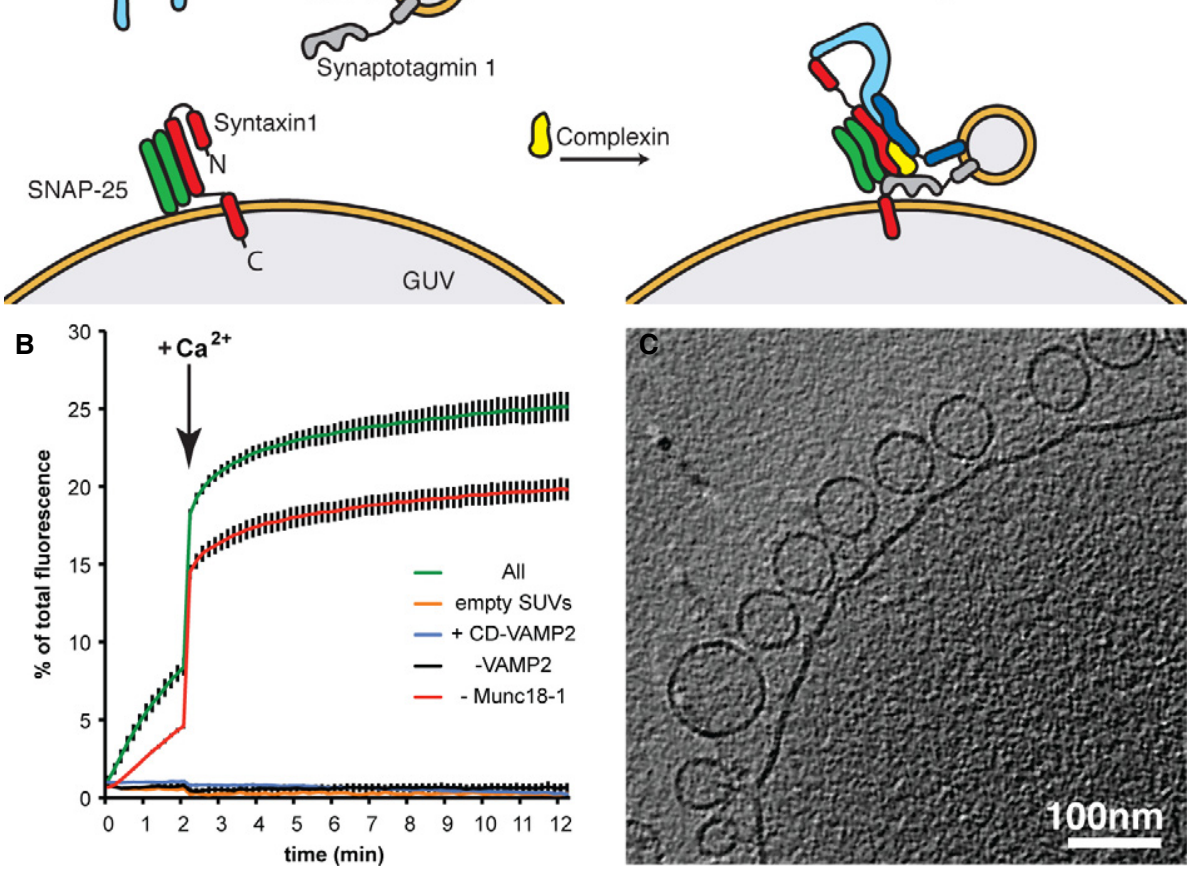

Figure 1. Observation of docked vesicles on the target membrane.

A A schematic representation of the in vitro system employed in this study.

B Kinetics of fusion reactions monitored by a plate reader assay for lipid mixing (see Supplementary Methods). Upon the addition of Ca ${ }^{2+}$, docked and primed vesicles fuse almost instantaneously as evident by a sudden increase in fluorescence signal indicating fast lipid mixing. Reaction mixtures contained or lacked the indicated components. All: all components (syntaxin1/SNAP-25, VAMP2, synaptotagmin 1, complexin, and Munc18) were present in the fusion assay; empty SUVs: protein-free SUVs lacking VAMP2 and synaptotagmin 1 were used; +CD-VAMP2: the cytoplasmic domain of VAMP2 was added to the reaction containing all components.

C Slice through a representative cryo-electron tomogram showing large populations of SUVs docked onto the target membrane in a reaction containing SNARE proteins, synaptotagmin 1, complexin and Munc18-1. Large populations of docked vesicles were not observed in a control reaction with empty SUVs. See also Supplementary Figs S1, S2A and Movie S1. 


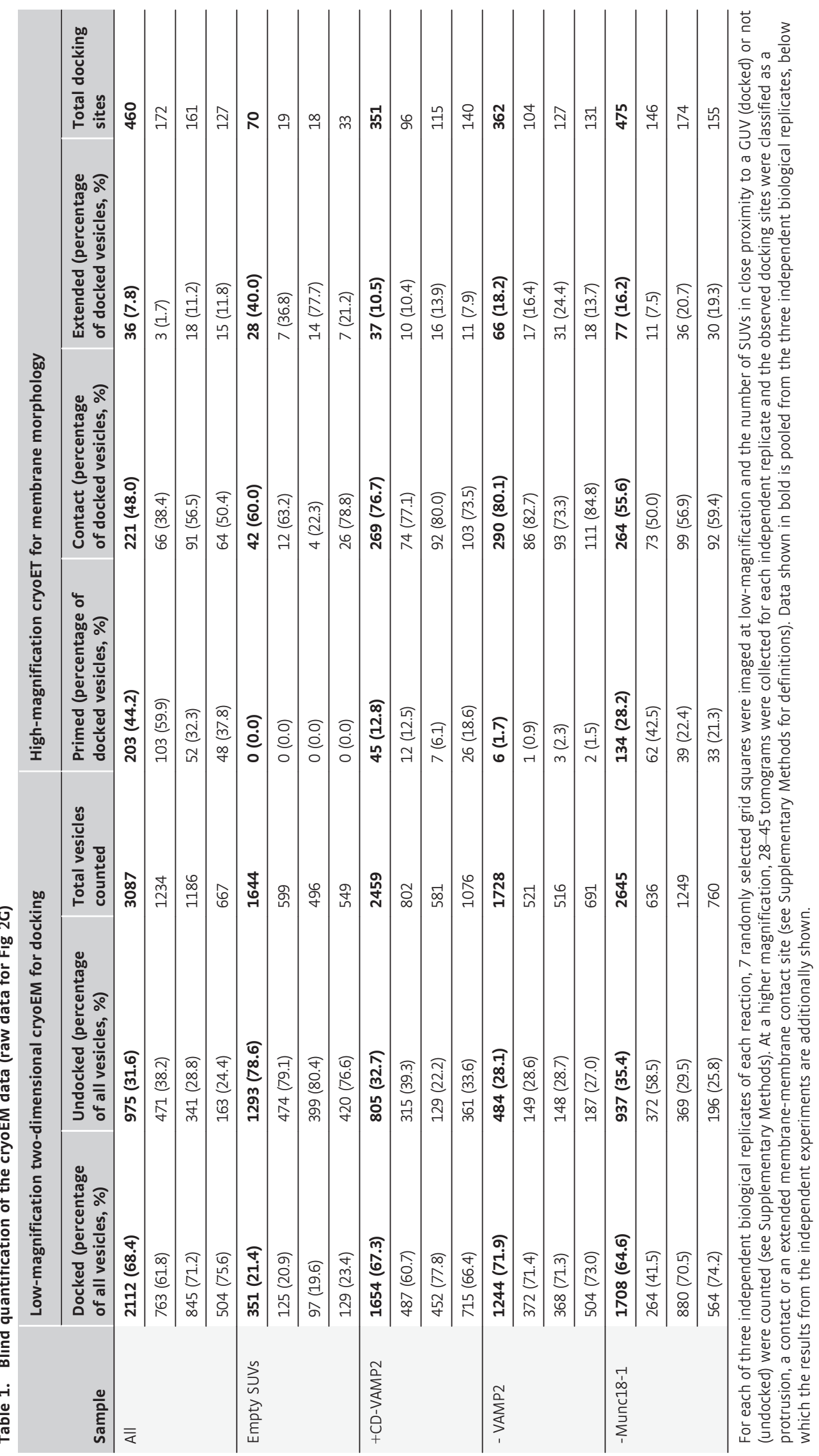


tomograms from the complete reaction showed large numbers of SUVs apparently docked to the GUV target membranes (Fig 1C, Supplementary Fig S2A, Movie S1). Quantitative analysis of overview images (Supplementary Fig S1) revealed that $68 \%$ of SUVs were proximal to a GUV in the complete reaction (Table 1), compared to only $21 \%$ of SUVs in control reactions where the SUVs contained no protein - these presumably represent SUVs in the vicinity of a GUV by random chance. Together these data suggest that approximately $69 \%$ of the SUVs that are proximal to GUVs in the complete reaction are specifically docked. In reactions in which VAMP2 was omitted, or in which the cytoplasmic domain (CD) of VAMP2 was added to compete with SNARE complex formation, or in which Munc18-1 was omitted, the fraction of docked vesicles was similar to that in the complete reaction, indicating that these components do not mediate or modulate docking (Table 1 and Fig 2G). In a control experiment in which synaptotagmin 1 was omitted, only a background level of docking was observed ( $\sim 8 \%$, Supplementary Table S3). This is consistent with previous studies indicating that synaptotagmin 1 is required to mediate vesicle docking $[14,16,23]$.

The high-magnification three-dimensional reconstructions of docked SUVs revealed three major classes of membrane morphology at the sites where a SUV was proximal to a GUV (Fig 2A-F, see Supplementary Methods for detailed definitions). In the first, simplest class ("contact") the SUV and GUV were in close proximity but there was no obvious distortion induced in the vesicle, or on the target membrane at the docking site (Fig $2 \mathrm{~B}$ and $\mathrm{E}$ ). The second class ("extended contact") was that of a more extended membranemembrane contact, with two closely apposing membranes (Fig 2C and F). In the third class ("protrusion") we observed a prominent protrusion in the GUV membrane at the point of closest approach, oriented towards the SUV (Fig S2A and D, Supplementary Fig S2B, Movie S1).

We hypothesized that these three classes represented different stages of membrane docking and fusion. In this case the addition or omission of regulatory components should alter the relative frequency of the morphologies observed. Thus, we prepared samples in which all components were present; where the cytoplasmic domain of VAMP2 (CD-VAMP2) was added as a competitor for trans-SNARE complex formation; where VAMP2 was absent; where VAMP2 and synaptotagmin 1 were absent ("empty SUVs"); and where Munc18-1 was absent. For each sample we used large-scale automated cryoET to reconstruct large numbers of docking sites in $3 \mathrm{D}$. The data for the different samples were mixed and randomized, and the occurrences of the different classes were quantified "blind". The experiment was repeated in triplicate for different biological replicates. The results of the quantification are summarized in Fig 2G (see also Table 1 and Supplementary Tables S1-S2, Supplementary Methods).

In the "empty SUV" control sample, where proximity between SUVs and GUVs presumably results from random chance, we found that $60 \%$ of sites showed a simple contact and $40 \%$ of sites showed an extended contact (Supplementary Fig S3). In this sample, the "protrusion" class was not observed. In contrast, in the complete reaction, $48 \%$ of sites showed a simple contact, $8 \%$ an extended contact, and $44 \%$ a protrusion. Considering that we estimated above that $69 \%$ of SUVs in the vicinity of the GUVs in the complete reaction are specifically docked, these data suggest that when all proteins are present, specific docking of SUVs induces a protrusion in the target membrane in the majority of cases.

To understand whether formation of the protruding membrane intermediate requires trans-SNARE complex assembly, we considered the sample where the cytoplasmic domain of VAMP2 was added as a competitor for t-SNARE binding. We observed that the number of protruding membrane intermediates observed was reduced by approximately $75 \%$ (Table 1 and Supplementary Tables S1-S2). In the sample where the SUVs were completely devoid of VAMP2 (containing only synaptotagmin 1), the effect was more dramatic: only $2 \%$ of docked SUVs showed a protrusion, $96 \%$ fewer than in the complete reaction. These experiments indicate that docking of vesicles to the target membrane, though mediated by synaptotagmin 1, is not sufficient to induce protrusions on the target membrane. (This observation rules out the possibility that protrusions are formed mechanically by docked SUVs being pulled away from the GUV membrane during sample preparation: in this case

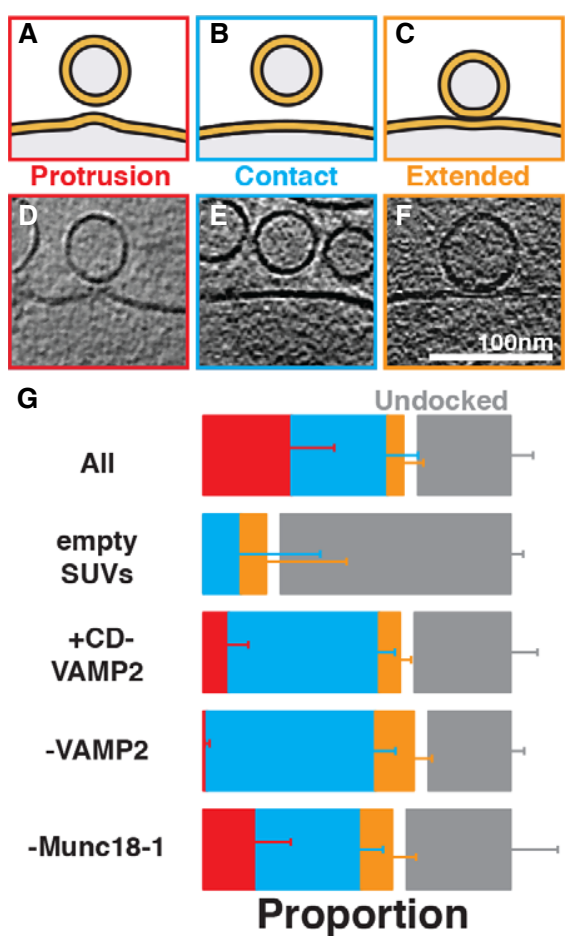

Figure 2. Large-scale automated cryoET and blind quantification of major membrane morphologies observed.

A-C Cartoons showing the three major types of membrane morphologies observed where SUVs were proximal to GUVs.

D-F Tomographic slices showing examples of $(A-C)$. See also Supplementary Fig S2B for a gallery of the protruding target membrane intermediate.

G Blind quantification (see Supplementary Methods) of the instances of each morphology observed in three independent biological replicates of each sample. Raw data are shown in Table 1. Error bars represent the $95 \%$ confidence intervals for each observed proportion (see Supplementary Methods and Supplementary Table S1-S2 for details of statistical analysis). The ratio of undocked (gray bar) to docked (red, blue and orange bars) vesicles was quantified using low-magnification images and the ratio of "protrusion", "contact" and "extended" contact vesicles was quantified using high-magnification cryoET data (see also Supplementary Figs S1, S2 and S3) 
one would expect protrusion formation to correlate with docking.) Formation of the protruding membrane intermediate is dependent on formation of a trans-SNARE complex.

The presence of Munc18-1 stimulated calcium-independent lipid mixing to a certain degree (Fig 1B), but it only resulted in a small increase in the formation of membrane protrusions, which was statistically not significant (Fig 2G, Supplementary Table S2). However, we cannot exclude that Munc18-1 may induce more subtle changes in the SUV or GUV membrane, which we did not score with our rigorous definition of protrusions.

We next asked whether at time points where priming was incomplete there was a corresponding lower number of protrusions. We therefore carried out an experiment in which a mixture of v-SNARE SUVs and t-SNARE GUVs was incubated on ice for different time periods in the presence of complexin. Subsequently we measured the extent of docking using a previously described liposome sedimentation assay [23] (Fig 3A, Supplementary Table S3), and measured the degree of fusion upon $\mathrm{Ca}^{2+}$ addition (Fig 3B-C). Further, we collected cryoET data (Fig 3D) at the different time points and "blindly" quantified the degree of protrusion formation. Combining these data, we found that between 1, 5, and 60 min time points, the amount of docking and of protrusion formation increased (Fig 3E). Simultaneously, the number of vesicles primed for fusion, as indicated by the extent of fusion upon $\mathrm{Ca}^{2+}$ addition, also increased (Fig 3C). Therefore, where priming was incomplete, a lower number of protrusions was observed.

This protruding target membrane intermediate was not observed in the recent cryoEM studies of SNARE mediated fusion in SUV mixtures in the absence of complexin $[25,26]$. In these studies either close contact between SUVs, or an extended contact region, was suggested to represent the pre-fusion intermediate. We hypothesize that protrusions are less easily visualized in SUVs than in GUVs due to the higher membrane curvature and membrane tension in the SUVs. We carried out an additional experiment where $\mathrm{v}$ - and t-SNARE proteins were each reconstituted into separate populations of SUVs that were then mixed in the presence of complexin and Munc18-1 and imaged by cryoEM and cryoET (Supplementary Fig S4A). Consistent with the recent studies, we did not observe the
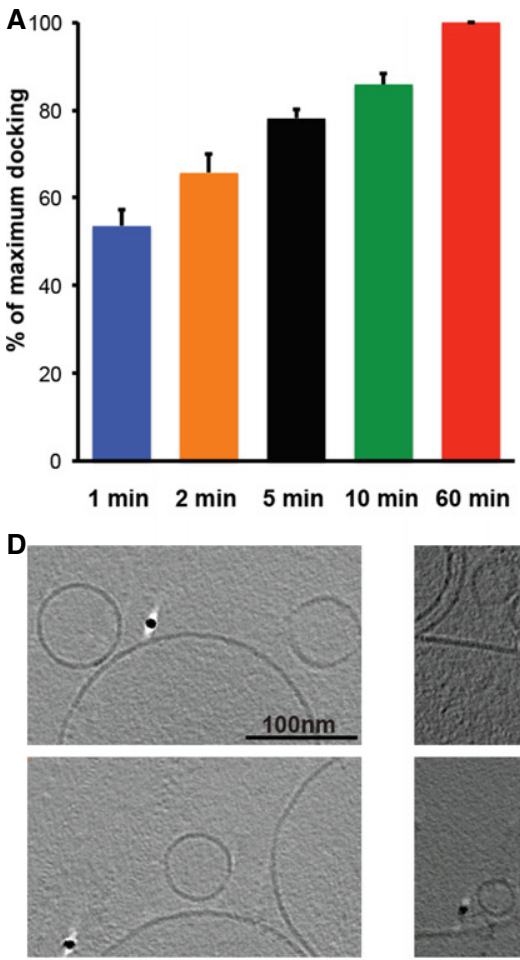

$1 \mathrm{~min}$
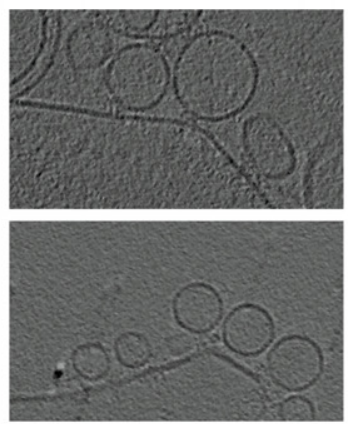

$5 \mathrm{~min}$
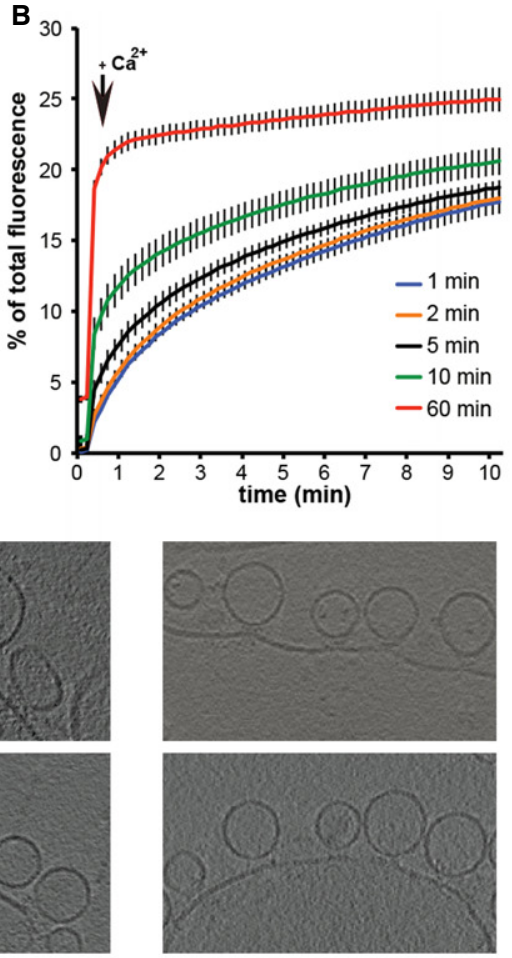

$60 \mathrm{~min}$

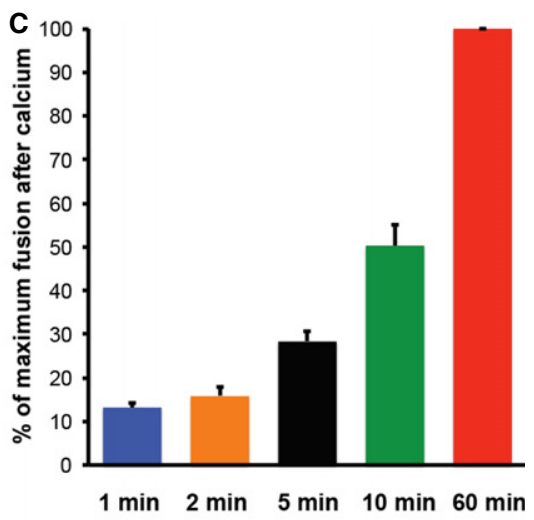

$\mathbf{E}$

$1 \mathrm{~min}$
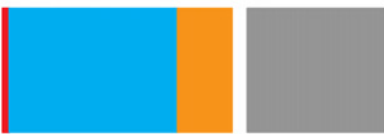

$5 \mathrm{~min}$

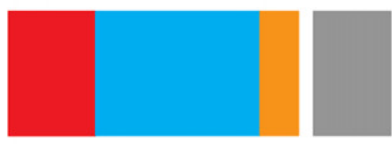

$60 \mathrm{~min}$

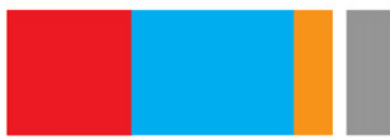

Figure 3. Protrusion formation correlates with priming and membrane fusion. Reactions containing VAMP2/synaptotagmin 1 SUVS were mixed on ice with t-SNARE GUVs in the presence of complexin, but in the absence of Munc18

A Rate of docking assayed by a liposome sedimentation assay described in [23] at different time points. Docking was found to increase as a function of time. Error bars indicate s.e.m. $(n=3)$.

B Kinetics of the fusion reaction at $37^{\circ} \mathrm{C}$ monitored by a plate reader assay for lipid mixing. Error bars indicate s.e.m. ( $n=3$ ).

C Change in fluorescence signal $10 \mathrm{~s}$ after induction with $\mathrm{Ca}^{2+}$ for the same samples. The extent of priming was seen to increase as a function of time. Error bars indicate s.e.m. $(n=3)$.

D Samples were prepared on ice and plunge-frozen for cryoEM at different time points at $4^{\circ} \mathrm{C}$. Representative tomographic slices from these experiments are shown ( $n=1$ for each time point).

E Quantification of randomized data from one sample at each time point (see Supplementary Methods) shows that both docking and protrusion formation increase as a function of time. Undocked vesicles (gray), contact (blue), extended contacts (orange), protrusions (red). 
formation of protrusions at contact sites between SUVs (Supplementary Fig S4A). We found that in this reaction, the similar sizes of the two SUV populations prevented discrimination of contacts between $\mathrm{v}$ - and t-SNARE containing SUVs from contacts between SUVs that both contain t-SNAREs or both contain v-SNAREs. We therefore repeated the reaction with all the components included (as in Fig 1C), but reconstituted the t-SNARE proteins into the SUVs and the v-SNARE proteins into the GUVs (Supplementary Fig S4C). Although fusion priming was efficient in this reaction (Supplementary Fig S4E), the formation of protrusions was inefficient (Supplementary Fig S4D, Table S3). These two observations together suggest that protrusion formation is specific to the t-SNARE-containing membrane, and is hindered by the high tension of SUVs.

In summary, we used a system where SUVs containing VAMP2 and synaptotagmin 1 (mimicking the small synaptic vesicles) were incubated with GUVs containing syntaxin1 and SNAP-25 (mimicking the low curvature of the synaptic plasma membrane), in the presence of complexin to stall the fusion reaction. We observed that SUVs efficiently dock with the GUVs to form an intermediate characterized by a local bilayer protrusion in the target membrane, directed towards the SUV. SUV docking was dependent on synaptotagmin 1 , but independent of trans-SNARE complex assembly. In contrast, induction of the membrane protrusion was dependent on formation of the trans-SNARE complex, which likely provides the energy required to deform the target membrane. Under conditions where fusion priming is incomplete, lower numbers of protrusions are seen. These data suggest that formation of a primed pre-fusion state is associated with a protruding membrane intermediate. Such protrusions have been hypothesized to be important for exocytosis [27]. Biophysical models have predicted that protein-mediated membrane fusion starts with a point-like contact between the vesicle and the target membrane, inducing a local lipid bilayer protrusion in the target membranes, followed by the formation of a hemi-fusion stalk and finally culminating in lipid bilayer merger $[28,29]$. Local bilayer protrusions have an increased membrane curvature, which can significantly reduce the kinetic barrier opposing spontaneous fusion within a biologically relevant time frame [30]. Based on our data, we propose that docked synaptic vesicles are primed in a high-energy state in which a local bilayer protrusion in the target membrane is stabilized by forces applied by the trans-SNARE complexes. In this primed state, activation with $\mathrm{Ca}^{2+}$ induces the insertion of the $\mathrm{C} 2$ domains of synaptotagmin 1 into the target membrane. This releases the complexin block, allowing the system to go energetically downhill towards full membrane fusion.

\section{Materials and Methods}

Protein reconstitution into liposomes, the assay for lipid-mixing, and sample preparation protocols for cryoEM have been described previously [14] (See also Supplementary Methods). The reaction partners were mixed and incubated for $1 \mathrm{~min}$ at $37^{\circ} \mathrm{C}$ before sample preparation for cryoEM, except for the time course assay for fusion (described in Fig 3) where cryoEM sample preparation was carried out at $4^{\circ} \mathrm{C}$. CryoEM data was collected on an FEI Polara microscope operated at $200 \mathrm{kV}$, equipped with an energy filter (see Supplementary Methods). Low-magnification overview images were collected with SerialEM [31], and cryoET data was collected using the FEI Tomography 4 software. CryoET data was processed using IMOD [32] and RAPTOR [33]. CryoEM data quantification was performed 'blind' by randomizing the data prior to image processing, unless otherwise specified. Statistical analysis is described in further detail in Supplementary Tables S1-S2 and in the Supplementary Methods section.

Supplementary information for this article is available online: http://embor.embopress.org

\section{Acknowledgements}

TAMB and JAGB would like to thank James D. Riches and Simone Prinz for facilitating initial cryoET data collection and Bernd Klaus and Hetty Manenschijn for supporting data analysis. The Briggs lab acknowledges financial support from EMBL and from the Chica und Heinz Schaller Stiftung. This work was supported by a grant of the German Research Foundation (SFB/TRR 83 to THS).

\section{Author contributions}

TAMB, JM, THS and JAGB designed experiments. TAMB, JM, AS performed experiments. WJHH supported large-scale cryoET data collection. TAMB, JM, THS and JAGB analyzed the data. TAMB, JM, THS and JAGB wrote the paper.

\section{Conflict of interest}

The authors declare no conflict of interest.

\section{References}

1. Bruns D, Jahn R (1995) Real-time measurement of transmitter release from single synaptic vesicles. Nature 377: 62-65

2. Sabatini BL, Regehr WG (1996) Timing of neurotransmission at fast synapses in the mammalian brain. Nature 384: 170-172

3. Rizo J, Rosenmund C (2008) Synaptic vesicle fusion. Nat Struct Mol Biol 15: $665-674$

4. Südhof TC (2004) The synaptic vesicle cycle. Annu Reu Neurosci 27: $509-547$

5. Sutton RB, Fasshauer D, Jahn R, Brunger AT (1998) Crystal structure of a SNARE complex involved in synaptic exocytosis at $2.4 \mathrm{~A}$ resolution. Nature 395: $347-353$

6. Fasshauer D, Sutton RB, Brunger AT, Jahn R (1998) Conserved structural features of the synaptic fusion complex: SNARE proteins reclassified as Q- and R-SNAREs. Proc Natl Acad Sci USA 95: 15781-15786

7. Söllner T, Whiteheart SW, Brunner M, Erdjument-Bromage H, Geromanos S, Tempst P, Rothman JE (1993) SNAP receptors implicated in vesicle targeting and fusion. Nature 362: 318-324

8. Stein A, Weber G, Wahl MC, Jahn R (2009) Helical extension of the neuronal SNARE complex into the membrane. Nature 460: 525-528

9. Gao Y, Zorman S, Gundersen G, Xi Z, Ma L, Sirinakis G, Rothman JE, Zhang Y (2012) Single reconstituted neuronal SNARE complexes zipper in three distinct stages. Science 337: 1340-1343

10. Südhof TC, Rizo J (2011) Synaptic vesicle exocytosis. Cold Spring Harb Perspect Biol 3: pii: a005637

11. Chapman ER (2008) How does synaptotagmin trigger neurotransmitter release? Annu Reu Biochem 77: 615-641

12. Jahn R, Fasshauer D (2012) Molecular machines governing exocytosis of synaptic vesicles. Nature 490: 201-207 
13. Martens S, McMahon HT (2011) C2 domains and membrane fusion. Curr Top Membr 68: 141-159

14. Malsam J, Parisotto D, Bharat TA, Scheutzow A, Krause JM, Briggs JA, Söllner TH (2012) Complexin arrests a pool of docked vesicles for fast $\mathrm{Ca}^{2+}$-dependent release. EMBO J 31: 3270-3281

15. Stein A, Radhakrishnan A, Riedel D, Fasshauer D, Jahn R (2007) Synaptotagmin activates membrane fusion through a $\mathrm{Ca}^{2+}$-dependent trans interaction with phospholipids. Nat Struct Mol Biol 14: 904-911

16. de Wit $\mathrm{H}$, Walter AM, Milosevic I, Gulyás-Kovács A, Riedel D, S1/2rensen JB, Verhage M (2009) Synaptotagmin-1 docks secretory vesicles to syntaxin-1/SNAP-25 acceptor complexes. Cell 138: 935-946

17. Reist NE, Buchanan J, Li J, DiAntonio A, Buxton EM, Schwarz TL (1998) Morphologically docked synaptic vesicles are reduced in synaptotagmin mutants of Drosophila. J Neurosci 18: 7662-7673

18. Wang Z, Liu H, Gu Y, Chapman ER (2011) Reconstituted synaptotagmin I mediates vesicle docking, priming, and fusion. J Cell Biol 195: $1159-1170$

19. Kim JY, Choi BK, Choi MG, Kim SA, Lai Y, Shin YK, Lee NK (2012) Solution single-vesicle assay reveals PIP2-mediated sequential actions of synaptotagmin-1 on SNAREs. EMBO J 31: 2144-2155

20. Maximov A, Tang J, Yang X, Pang ZP, Südhof TC (2009) Complexin controls the force transfer from SNARE complexes to membranes in fusion. Science 323: $516-521$

21. Kümmel D, Krishnakumar SS, Radoff DT, Li F, Giraudo CG, Pincet F, Rothman JE, Reinisch KM (2011) Complexin cross-links prefusion SNAREs into a zigzag array. Nat Struct Mol Biol 18: $927-933$

22. Xu Y, Su L, Rizo J (2010) Binding of Munc18-1 to synaptobrevin and to the SNARE four-helix bundle. Biochemistry 49: 1568-1576

23. Parisotto D, Malsam J, Scheutzow A, Krause JM, Söllner TH (2012) SNAREpin assembly by Munc18-1 requires previous vesicle docking by synaptotagmin 1. J Biol Chem 287: 31041-31049
24. Vrljic M, Strop P, Ernst JA, Sutton RB, Chu S, Brunger AT (2010) Molecular mechanism of the synaptotagmin-SNARE interaction in $\mathrm{Ca}^{2+}$-triggered vesicle fusion. Nat Struct Mol Biol 17: $325-331$

25. Hernandez JM, Stein A, Behrmann E, Riedel D, Cypionka A, Farsi Z, Walla PJ, Raunser S, Jahn R (2012) Membrane fusion intermediates via directional and full assembly of the SNARE complex. Science 336: $1581-1584$

26. Diao J, Grob P, Cipriano DJ, Kyoung M, Zhang Y, Shah S, Nguyen A, Padolina M, Srivastava A, Vrljic M, Shah A, Nogales E, Chu S, Brunger AT (2012) Synaptic proteins promote calcium-triggered fast transition from point contact to full fusion. Elife 1: e00109

27. Monck JR, Fernandez JM (1992) The exocytotic fusion pore. J Cell Biol 119: $1395-1404$

28. Efrat A, Chernomordik LV, Kozlov MM (2007) Point-like protrusion as a prestalk intermediate in membrane fusion pathway. Biophys J 92: L61-L63

29. Chernomordik LV, Kozlov MM (2008) Mechanics of membrane fusion. Nat Struct Mol Biol 15: 675-683

30. Kozlov MM, McMahon HT, Chernomordik LV (2010) Protein-driven membrane stresses in fusion and fission. Trends Biochem Sci 35: $699-706$

31. Mastronarde DN (2005) Automated electron microscope tomography using robust prediction of specimen movements. J Struct Biol 152: $36-51$

32. Kremer JR, Mastronarde DN, McIntosh JR (1996) Computer visualization of three-dimensional image data using IMOD. J Struct Biol 116: $71-76$

33. Amat F, Castaño-Diez D, Lawrence A, Moussavi F, Winkler H, Horowitz M (2010) Alignment of cryo-electron tomography datasets. Methods Enzymol 482: 343-367 